\title{
Design and Engineering Application of Polymer Grouting Pore Layout Scheme for Expressway Pavement Diseases
}

\author{
Liu Tao, Zheng Zhigang, Hong Shaoyou, Huang Zhi, Wu Yintan, Huang Hong \\ Shenzhen Yuetong Construction Engineering Co., Ltd
}

\begin{abstract}
In grouting technology, after choosing the type of grouting fluid needed for grouting, the next most important step is the layout of grouting holes, that is, grouting hole type and hole spacing. Reasonable arrangement of grouting holes and hole spacing can not only achieve the desired reinforcement effect, but also save unnecessary economic expenses. For this reason, the effective diffusion radius of slurry is firstly determined considering both the characteristics of polymer slurry and the pore structure of injected medium. Based on this, the distance between holes (triangular and rectangular) is determined. Finally, the grouting design parameters of this design scheme are applied to the disease area of Nanping Expressway in Shenzhen. The deflection value before and after grouting is detected by falling weight deflectometer (FWD). The results show that the grouting design parameters can achieve better grouting effect.
\end{abstract}

\section{Introduction}

With the rapid development of China's national economy, China's highway construction is also springing up. By the end of 2018, China's mileage in highway open to traffic has reached 4.8465 million kilometers, ranking first in the world. However, China's highway construction started late, with limited technical level and maintenance experience, and most of the subgrade and pavement have diseases which are manifested by the road cracking, void, faulting of slab ends, deflection and mud pumping caused by complex engineering geological conditions, poor construction quality and vehicle overload. Most of these diseases appear in the local sections of the highway. When the traditional methods of excavation, refueling and backfilling are used, the cost is high and the construction period is long. Moreover, the secondary diseases are easy to occur due to the poor coordination between the new section and the original subgrade and pavement, so it is difficult to completely eliminate the hidden dangers.

Polymer grouting technology is a rapid maintenance technology developed in the 1970s [1-3]. It is mainly used for building foundation reinforcement or floor repair in foreign countries. Under the research of Zhengzhou University and other units in China, nondestructive testing technology and polymer grouting technology have been organically integrated, and a complete set of rapid maintenance polymer grouting technology based on nondestructive testing technology has been developed. It is widely used in fast trenchless maintenance and reinforcement of road, bridge, dam, tunnel and building foundation. Because it can be applied locally, it shows great construction and cost advantages. However, in the treatment of subgrade subsidence, there are still some problems such as single grouting method, inaccurate grouting positioning and poor grouting effect.

The spatial layout of grouting holes (hole type and hole spacing) plays a significant role in the process of grouting treatment of engineering diseases [4-7]. Reasonable space layout of grouting holes can not only achieve good grouting effect, but also greatly save the cost of disease control. Therefore, in the process of disease grouting treatment, the optimization design and selection of type and spacing parameters of grouting hole is another important factor to be considered besides the type of grout.

In this paper, starting from the mechanism of grouting reinforcement of highway subgrade, the theoretical problems of spatial layout of grouting hole, grouting diffusion radius and grouting amount are studied, so as to provide reliable theoretical basis for the selection of grouting design parameters of highway subgrade disease treatment.

\section{Diffusion radius of a single grouting hole}

According to literature [7], the diffusion pressure and diffusion radius of slurry can be expressed as:

$$
\begin{aligned}
& -\frac{\lambda_{\max }^{1+D_{\mathrm{T}}} \phi\left(2-D_{\mathrm{f}}\right) \mathrm{d} p}{32 \mu L^{D_{T}-1} D_{T}\left(3+D_{T}-D_{\mathrm{f}}\right) \mathrm{d} L} /\left[1-\left(\frac{\lambda_{\min }}{\lambda_{\max }}\right)^{2-D_{\mathrm{f}}}\right] \\
& -\frac{\lambda_{\max } \tau_{0} \phi\left(2-D_{f}\right)}{8 \mu\left(3-D_{f}\right)} /\left[1-\left(\frac{\lambda_{\min }}{\lambda_{\max }}\right)^{2-D_{\mathrm{f}}}\right]-\sqrt[6]{\frac{3 Q}{4 \pi t^{3}}}=0
\end{aligned}
$$

where $\lambda_{\max }$ and $\lambda_{\min }$ are the maximum radius and the minimum radius of the hole channel respectively; $\phi$ is the porosity of the injected medium; $D_{f}$ is the fractal

\footnotetext{
* Corresponding author: 315778102@qq.com
} 
dimension of holes; $D_{T}$ is the fractal dimension of hole tortuosity; $\mu$ is the viscosity of the slurry; $\tau_{0}$ is the initial shear force; $t$ is the grouting time; $Q$ is the grouting volume.

\section{Analysis of type and spacing of grouting hole}

The optimization of type and spacing of grouting hole is the most important way to ensure the grouting effect. This is because the grouting hole type can determine the diffusion distribution of the slurry, with the commonly used layout methods of grouting hole, which mainly include rectangular type, plum blossom type and array type [8-10], among which the rectangular type and plum blossom type are the most commonly used. Moreover, the grouting hole spacing mainly involves the problems of blind area of slurry diffusion and the mutual interference of slurry diffusion. For example, when the grouting hole spacing is small, it will cause the slurry interference between adjacent grouting holes, and it will increase the cost of disease control due to the layout of too many grouting holes. When the grouting hole spacing is large, it is easy to produce the blind area caused by the uneven slurry diffusion. Therefore, the optimization of type and spacing of grouting hole plays an important role in the treatment of the disease grouting.

\subsection{Layout of triangular (plum blossom) hole type}

The schematic diagram of triangular hole layout is shown in Figure 1. In order to facilitate the study, it is assumed that the effective diffusion radius of each grouting hole is the same in this paper. According to reference [7], when the confluence of the three grouting holes is located at the center of the circumscribed circle of the triangle, the blind area of grouting diffusion of the three holes is the smallest. According to the sine theorem, the effective diffusion radius of the corresponding grouting hole is obtained as:

$$
R_{1}=\frac{\sqrt{3}}{3} a=\frac{\sqrt{3}}{3} \mathrm{~b}=\frac{\sqrt{3}}{3} c
$$

Then, it can be obtained that the transverse distance of adjacent grouting holes is $a=\sqrt{3} R_{1}$, and the longitudinal distance is $h=\sqrt{3} a / 2=1.5 R_{1}$.

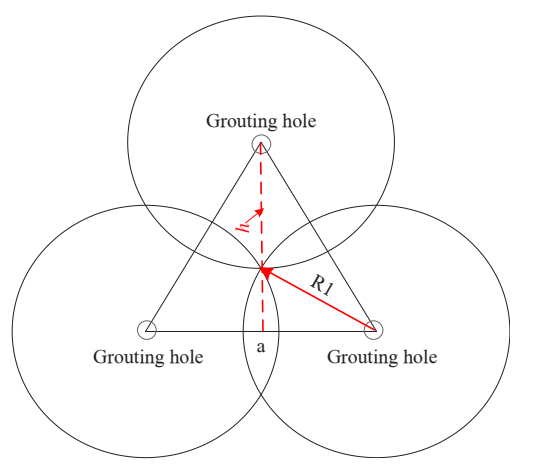

Fig. 1. Schematic diagram of triangular hole layout

\subsection{Layout of triangular (plum blossom) hole type}

The schematic diagram of rectangular hole layout is shown in Figure 2. In order to facilitate the study, it is assumed that the effective diffusion radius of each grouting hole is the same in this paper. According to reference [7], when the confluence of the four grouting holes is located at the center of the circumscribed circle of the rectangle, the blind area of grouting diffusion of the four holes is the smallest. The effective diffusion radius of the corresponding grouting hole can be obtained as:

$$
H=\sqrt{\left(\frac{a}{2}\right)^{2}+\left(\frac{b}{2}\right)^{2}}=\frac{\sqrt{2}}{2} a
$$

Then, it can be obtained that the transverse distance of adjacent grouting holes is $a=\sqrt{2} R_{1}$, and the longitudinal distance is $b=\sqrt{2} R_{1}$.

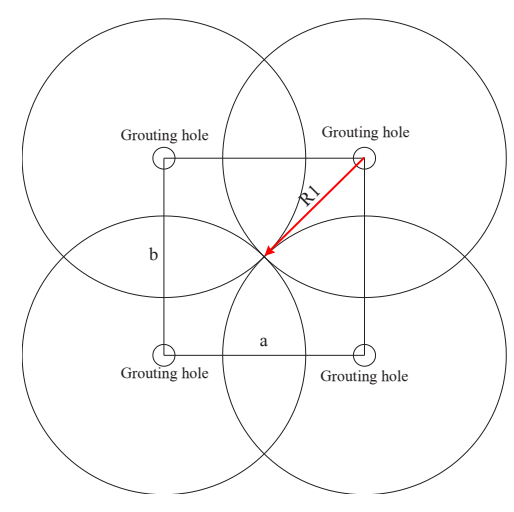

Fig. 2. Schematic diagram of rectangular hole layout

\section{Engineering application}

Nanping Expressway has been built for ten years, with huge traffic flow and numerous trucks. Under the repeated action of heavy load for a long time, the quality of the pavement has been declining year by year, and the base course of some sections has been seriously damaged and cracked and uneven settlement also appears, resulting in structural rutting, sinking, cracking, loosening, pumping, potholes and peeling of the surface course, as shown in Figure 3. In particular, the cement stabilized granular base of the third and fourth lanes of Nanping eastbound K7+890 has been cracked, loose, wet and soft, and there is a severe phreatic phenomenon, and there is no condition for drainage of base water around. Due to the insufficient bearing capacity of the base course, there are many defects such as potholes, mud pumping and so on, which cannot be completely cured by common milling and resurfacing of the asphalt on the surface course. 

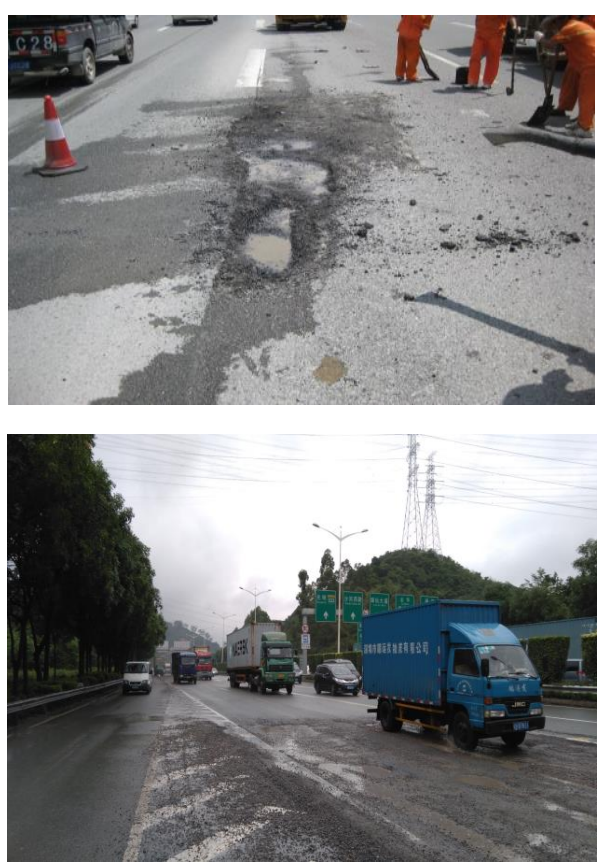

Fig. 3. NDJ-5T digital rotational viscometere

\subsection{Layout of triangular (plum blossom) hole type}

In order to obtain better grouting effect, it is necessary to select precise grouting design parameters. Therefore, this paper makes a quantitative study on the characteristic parameters of the hole structure of the medium in the pre-injection area. Through drilling and coring in the disease area of Nanping Expressway in Shenzhen, the particle size distribution characteristics of the medium in the injected area are shown in Figure 4, and then the characteristic parameters of the hole structure of the injected medium are obtained, as shown in Table 3.

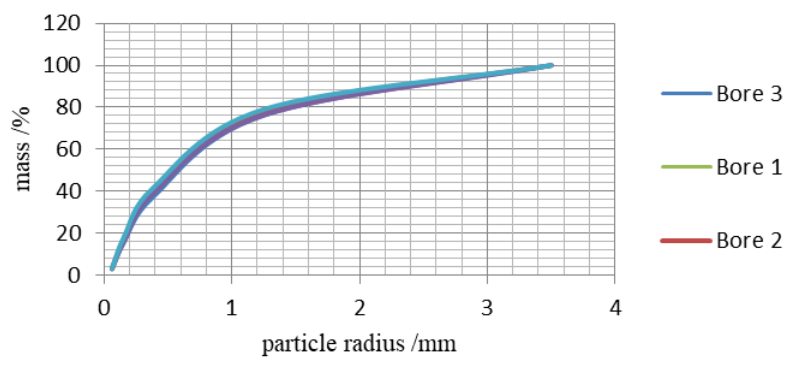

Fig. 4. Particle size distribution

Table 1 Hole characteristic parameters of injected medium

\begin{tabular}{|c|c|c|c|c|c|c|}
\hline & $\begin{array}{c}\text { Uniformity } \\
\text { coefficient } C \\
u\end{array}$ & $d_{50}$ & $\lambda_{\min } / \lambda_{\max }$ & $D_{T}$ & $D_{f}$ & $\begin{array}{c}\rho \\
\mathrm{g} \cdot \mathrm{cm}^{-3}\end{array}$ \\
\hline 1 & 6.8 & 0.86 & 0.0698 & 1.11 & 1.81 & 1.934 \\
\hline 2 & 7.0 & 0.84 & 0.0673 & 1.12 & 1.80 & 1.984 \\
\hline 3 & 7.1 & 0.85 & 0.0684 & 1.12 & 1.79 & 1.967 \\
\hline
\end{tabular}

Note: Hole characteristic parameters $\left(D_{T}, D_{f}, \lambda_{\min }\right.$ and $\left.\lambda_{\max }\right)$ are obtained by reference [11-14]

\subsection{Fluid characteristics of polymer slurry}

The material used in polymer grouting technology is non-aqueous two-component foaming polyurethane. Polyurethane material is a kind of high polymer material made of polyisocyanate and polyol, which is usually called high polymer material. Compared with the original grouting material, it has the advantages of good grouting performance, short reaction time, good antiseepage performance, and it can change the performance according to the needs of the project. It is an ideal grouting material. The fluid characteristic parameters are shown in Table 2.

Table 2 Rheological parameters and density of polymer slurry

\begin{tabular}{|c|c|c|c|}
\hline type & $\begin{array}{c}\text { Density }\left(\mathrm{g} \cdot \mathrm{cm}^{-}\right. \\
\left.{ }^{2}\right)\end{array}$ & $\begin{array}{c}\text { viscosity } \\
(\mathrm{Pa} \cdot \mathrm{s})\end{array}$ & $\begin{array}{c}\text { Gelling time } \\
(\mathrm{s})\end{array}$ \\
\hline Polymer & 0.21 & 2.2 & 60 \\
\hline
\end{tabular}

\subsection{Layout of grouting hole}

It can be seen from GPR that the disease area is not evenly distributed. Therefore, in this paper, the triangular hole layout mode is adopted, and the corresponding hole spacing parameters can be obtained through formula (1) in combination with table 1 and table 2 , as shown in Table 3.

Table 3 Rheological parameters and density of polymer slurry

\begin{tabular}{|c|c|c|}
\hline Hole pattern & $\begin{array}{c}\text { Radius } / \mathrm{m} \\
\mathrm{R}(p)\end{array}$ & Hole distance $/ \mathrm{m}$ \\
\hline Triangle & 1.1 & $(\mathrm{a}=1.90 ; \mathrm{h}=1.65)$ \\
\hline
\end{tabular}

\subsection{Layout of grouting hole}

The FWD (falling weight deflectometer) lifts and drops a heavy hammer through the hydraulic system under the control of computer to apply pulse load to the road surface, as shown in Figure 5. The magnitude of the load can be adjusted in a large range by changing the weight of the hammer and the lifting height, and the load is applied to the road surface through the rigid disk. The deformation of pavement is measured by several sensors.

FWD is considered to be an ideal non-destructive testing equipment for pavement because of its high test speed and accuracy, as well as its good simulation of the dynamic effect of traffic load. In particular, FWD can accurately measure multi-point deflection, which provides a basis for the inverse analysis of pavement structure.

One day after the grouting, FWD (Dynatest 8000) was used to detect the above disease areas, and the grouting effect was obtained by comparing and analyzing the deflection difference before and after grouting. 


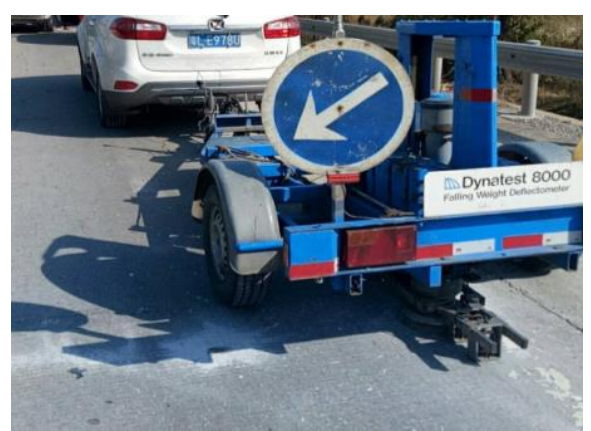

Fig. 5. Falling weight deflectometer (FWD)

FWD test results show that the deflection values are reduced in different degrees after grouting. Figure 6 shows the comparison curve of the deflection changes before and after grouting at each measuring point in the disease area of Nanping Expressway in Shenzhen. It can be seen that the average deflection values of the measuring points after grouting are reduced by $54 \%$, which shows that the bearing capacity of the pavement is effectively improved by high polymer grouting. In addition, after the high polymer grouting treatment, the phenomenon of mud pumping at each crack has been significantly improved. It can be obtained from the observation of rain and spring thawing period taht there is no mud pumping after the maintenance of the pavement with polymer grouting.

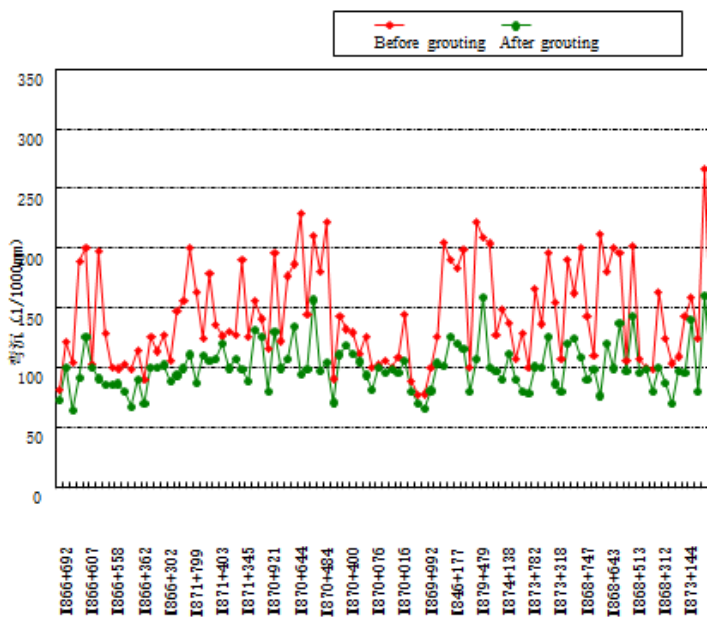

Fig. 6. Law diagram of falling weight deflectometer (FWD)

\section{Conclusions}

(1) The optimal design and calculation method for the selection of hole type and hole spacing in the process of grouting treatment of pavement diseases are obtained.

(2) Considering both the characteristics of polymer slurry and the hole structure of the injected medium, the effective grouting radius obtained is closer to the reality, which provides technical support for the precise layout of subsequent multi-hole grouting and has better engineering application value.

(3) FWD is used to detect the deflection value of the road before and after grouting, and the result shows that the grouting scheme can solve the disease of the disease area well.

\section{References}

1. C.C. Guo, B. Sun, D.P. Hu, Soil Mech. Foundation Eng, 56, 7 (2019).।

2. X.P. Liu, Transpoworld, 35, 3 (2018)

3. F.M. Wang, W.H. Li, C.C. Guo, J. Beijing jiaotong univ, 437 (2019)

4. Z.P. Li, D.M. Wang, L. Zhang, Const. technol 48, 7 (2019)

5. W.R. He, F.L. He, K. Zhang, J. min. Sci. technol 4, 9 (2019)

6. J. Zhao, J.F. Zou S. Zeng, J. Cent. South Univ 41, 5 (2010)

7. Z.L. Zhou, X.M. Du, S.Y. Wang, Constr. Build. Mater 186, 13 (2018)

8. J. Wei, Spirtual leaders (first and middle) 11, 2 (2014)

9. X. Chen, H.Z. Xiao, Bridge Constr 5, 3 (2010)

10. Z.L. Zhou, Y.L. Zhao, Z. Chen, J. Cent. South Univ. 48, 8 (2017)

11. Z.L. Zhou, X.M. Du, Z. Chen, The Chin. J. Nonferrous Metals 26, 7 (20168)

12. Z.L. Zhou, X.M. Du, Z. Chen, J. Cent. South Univ 24, 7 (2017)

13. F. Zhu, J. Power Sour 243,4 (2013)

14. Q. Zheng, B. Yu, Y. Duan, Chem. Eng. Sci 87, 7 (2013) 